\title{
Terahertz Spectroscopy for Characterization of Hydrogen Bonding and Cross-linked Structure Dynamics in Polyurethane
}

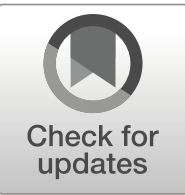

\author{
Hiromichi Hoshina ${ }^{1}$ (D) Yuichi Saito ${ }^{1} \cdot$ Takumi Furuhashi $^{2} \cdot$ Tomomi Shimazaki $^{2}$. \\ Manabu Sawada ${ }^{2} \cdot$ Yasunori Hioki $^{2} \cdot$ Chiko Otani $^{1}$
}

Received: 4 October 2019 / Accepted: 25 December 2019 / Published online: 4 January 2020

(C) The Author(s) 2020

\begin{abstract}
Terahertz (THz) absorption spectra of cross-linked polyurethane films were observed at 30-180 ${ }^{\circ} \mathrm{C}$. The obtained spectra displayed characteristic absorption bands. A band observed at $2.6 \mathrm{THz}$ was assigned to the $\mathrm{C}=\mathrm{O} \cdots \mathrm{N}-\mathrm{H}$ hydrogen bond vibration using previously reported data. DFT calculation results confirmed that a band at $7.5 \mathrm{THz}$ arises owing to the torsional vibration of cross-linked molecules containing urethane bonds. The slight changes in the temperature-dependent spectra were analyzed by the first derivative of absorption intensity with temperature. The thermal behavior of absorption intensities indicates the dynamics of hydrogen bonds and cross-linked structures in polyurethane films.
\end{abstract}

Keywords Terahertz spectroscopy $\cdot$ Polymer $\cdot$ Hydrogen bond $\cdot$ Polyurethane $\cdot$ Cross-linked structure

\section{Introduction}

Because terahertz $(\mathrm{THz})$ frequencies are significantly lower than infrared (IR) light frequencies, absorption features in the $\mathrm{THz}$ region can be mainly attributed to the vibrations of weaker intermolecular interactions (e.g., hydrogen bonds or van der Waals interactions) in massive molecules, such as molecular aggregates or macromolecular structures $[1,2]$. THz spectroscopy can be used to elucidate higher order structures within macromolecules. Absorption

Electronic supplementary material The online version of this article (https://doi.org/10.1007/s10762-01900667-0) contains supplementary material, which is available to authorized users.

Hiromichi Hoshina

hoshina@ riken.jp; https://orcid.org/0000-0002-4770-2005

1 RIKEN Center for Advanced Photonics, Sendai, Miyagi 980-0845, Japan

2 Murata Manufacturing Co., Ltd., Nagaokakyo, Kyoto 617-8555, Japan 
frequencies and intensities provide rich information about the conformation of higher order structures, which imparts macromolecular properties [3-6]. Therefore, THz spectroscopy is expected to become a novel tool to characterize macromolecular structure and dynamics.

In our previous studies, we observed $\mathrm{THz}$ spectra of polymers with hydrogen bonds. Based on the THz polarization spectra and low-frequency Raman spectra, the vibrational modes of crystalline polymers were determined [6-10]. In addition, we developed a computational method to enable the precise assignment of each vibrational mode [8-10]. As a result, we determined that polymers having similar structures exhibit comparable absorption features in their THz spectra. For example, poly(hydroxybutyrate) displays an intense absorption band at 2.5 THz, which is assigned to the coupled bending motion of $\mathrm{C}=\mathrm{O}$ and $\mathrm{CH}_{3}$ [8]. A similar vibrational band (bending motion of $\mathrm{C}=\mathrm{O}$ and $\mathrm{CH}_{2}$ ) is observed in the spectrum of poly(glycolic acid) at $2.7 \mathrm{THz}$ [9]. These modes are characteristic of hydrogen bond between $\mathrm{C}=\mathrm{O}$ and $\mathrm{CH}_{\mathrm{x}}$. Importantly, the vibration frequency red shifts at higher temperatures due to changes in hydrogen bond lengths caused by thermal expansion $[7,9,10]$.

Furthermore, characteristic bands are found in polymers with $\mathrm{C}=\mathrm{O} \cdots \mathrm{N}-\mathrm{H}$ hydrogen bonds. It has been reported that polyamides exhibit intense absorption bands at approximately $3 \mathrm{THz}$ [11-15]. Recently, the bands observed at $3.2 \mathrm{THz}$ in the spectrum of nylon- 6 were assigned to the coupled bending motion of $\mathrm{C}=\mathrm{O}, \mathrm{N}-\mathrm{H}$, and neighboring $\mathrm{CH}_{2}$ using DFT calculations [10]. The characteristic band for a $\mathrm{C}=\mathrm{O} \cdots \mathrm{N}-\mathrm{H}$ hydrogen bond is also observed at $3.4 \mathrm{THz}$ in organic gels containing $\mathrm{C}=\mathrm{O} \cdots \mathrm{N}-\mathrm{H}$ hydrogen bonds [16]. The frequency of the band reflects the supramolecular structure of the gel; the frequency drastically changes with a sol-gel phase transition [16]. Polyurethane also contains $\mathrm{C}=\mathrm{O} \cdots \mathrm{N}-\mathrm{H}$ hydrogen bonds. Zavodov et al. reported absorption bands around $100 \mathrm{~cm}^{-1}(\sim 3 \mathrm{THz})$ in the far-IR spectra of all samples of segmented polyurethane [17].

In this study, the $\mathrm{THz}$ spectra of cross-linked polyurethane films, a thermosetting plastic used for electric devices, are determined at different temperatures. The cross-linked structure comprises urethane bonding, in which the $\mathrm{C}=\mathrm{O}$ functional group is included in $\mathrm{C}=\mathrm{O} \cdots \mathrm{N}-\mathrm{H}$ hydrogen bonds. Macromolecular structures and their dynamics are discussed according to the frequencies and intensities observed in $\mathrm{THz}$ absorption spectra.

\section{Materials and Methods}

In this study, two different polyurethane films were evaluated - samples A and B. Figure 1 shows the macromolecular structure of cross-linked polyurethane and the molecular structures of samples A and B. Poly(hydroxy ether) of bisphenol-A (BPAP) and phenoxy copolymer from bisphenol-TMC and TMDHB (BP-TMC-co-TMDHB) were used as the main agents for samples $\mathrm{A}$ and $\mathrm{B}$, respectively; 4,4'-diphenylmethane diisocyanate (MDI) was used as the curing agent.

To construct a film sample, a two-step process was used. First, a 70:30 (wt\%) ratio of main agent to curing agent was mixed and dissolved in methyl ethyl ketone (MEK) at $20 \mathrm{wt} \%$. Next, the mixture was spread onto a polyethylene terephthalate (PET) film to form a film with the thickness of $3 \mu \mathrm{m}$, the typical thickness used for electric devices. The film was warmed to $60{ }^{\circ} \mathrm{C}$ over $20 \mathrm{~h}$, and then was maintained at $60{ }^{\circ} \mathrm{C}$ for $20 \mathrm{~h}$. Under these conditions, approximately $50 \%$ cross-linked structure was formed. Films were then removed from the PET films, stacked to 100 sheets, and heat-pressed at $73.4 \mathrm{MPa}$ and $80{ }^{\circ} \mathrm{C}$ for $5 \mathrm{~min}$ to form a single film with a thickness of $250 \mu \mathrm{m}$, which is appropriate for $\mathrm{THz}$ measurements. To complete the cross-linking reaction, the samples were gradually heated to $180{ }^{\circ} \mathrm{C}$ over $1 \mathrm{~h}$, and then maintained at $180^{\circ} \mathrm{C}$ for $4 \mathrm{~h}$. 


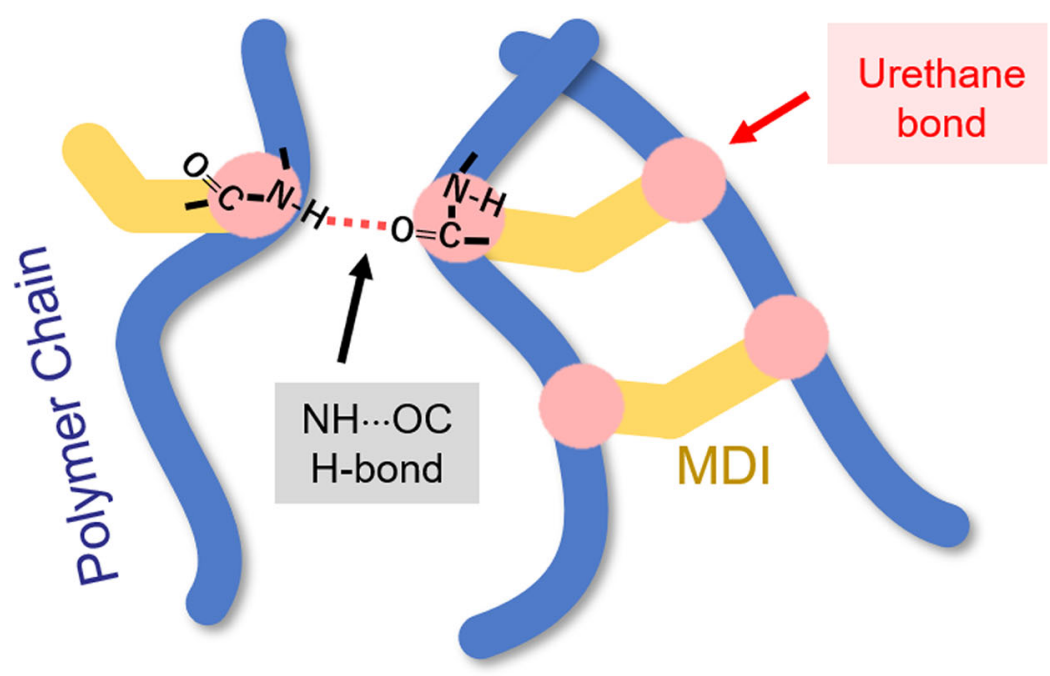

Sample A

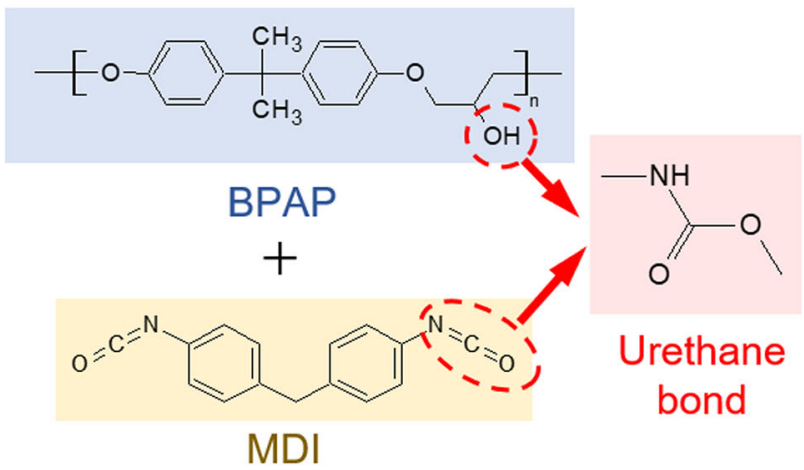

\section{Sample B}

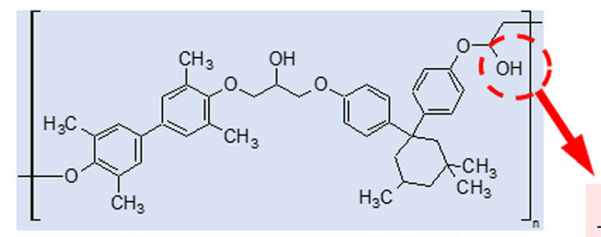

BP-TMC-CO-TMDHB

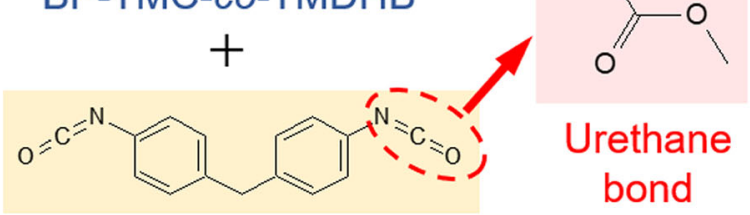

MDI

Fig. 1 Macromolecular structure of cross-linked polyurethane and molecular structures of samples A and B 
Sample crystallinity was assessed using wide angle X-ray diffraction; no peak was observed. The macromolecular structure of the sample is amorphous. The glass transition temperature, $T_{g}$, measured by differential scanning calorimetry, was $175{ }^{\circ} \mathrm{C}$ for sample A and $245^{\circ} \mathrm{C}$ for sample B.

$\mathrm{THz}$ absorption spectra were observed using Fourier-transform far-IR spectrometer (FTFIR) (JASCO: FARIS) combined with a superconducting bolometer (QMC: QNbB/PTC), which detects $\mathrm{THz}$ frequencies with higher sensitivity and stability than those of conventional bolometers. A high-pressure mercury lamp was used as the light source and a wire-grid beam splitter was used for measurements with a resolution of $0.06 \mathrm{THz}$. A total of 200 scans were accumulated in $3 \mathrm{~min}$ to obtain a single spectrum. The schematics of the heated sample holder are shown in Fig. 2. The sample film was sandwiched between a pair of aluminum holders and set to Brewster's angle, $\theta_{B}$, to reduce multiple internal reflections (to avoid fringe in the absorption spectrum) [18]. Brewster's angle was set to $55^{\circ}$ using the typical index of refraction for polymers $\sim 1.5$ [5]. The THz beam was linearly polarized using a wire-grid polarizer. The temperature of the sample holder was changed from 30 to $180^{\circ} \mathrm{C}$ by controlling the rubber heater current using a PC running a PID program, which monitors temperature using a Pt-100 sensor. Prior to measurements, the temperature difference between the sample and Pt-100 sensor was confirmed. The difference of the temperature was less than $3{ }^{\circ} \mathrm{C}$ for the temperature range measured. For convenience, the value of Pt-100 is denoted as "temperature" herein.

\section{Results and Discussions}

Figure 3 shows the absorption and second derivative spectra of samples A and B measured at $30^{\circ} \mathrm{C}$. In this paper, smoothing is applied for all the absorption spectra to remove spike noise originated in the absorption of the residual water vapor in the sample chamber. The spectra were averaged by Origin $^{\mathrm{TM}}$ software with adjacent average option with 100 points $(\sim 0.7 \mathrm{THz})$, which does not "over-

Fig. 2 Schematics of the heating sample holder with Brewster's angle

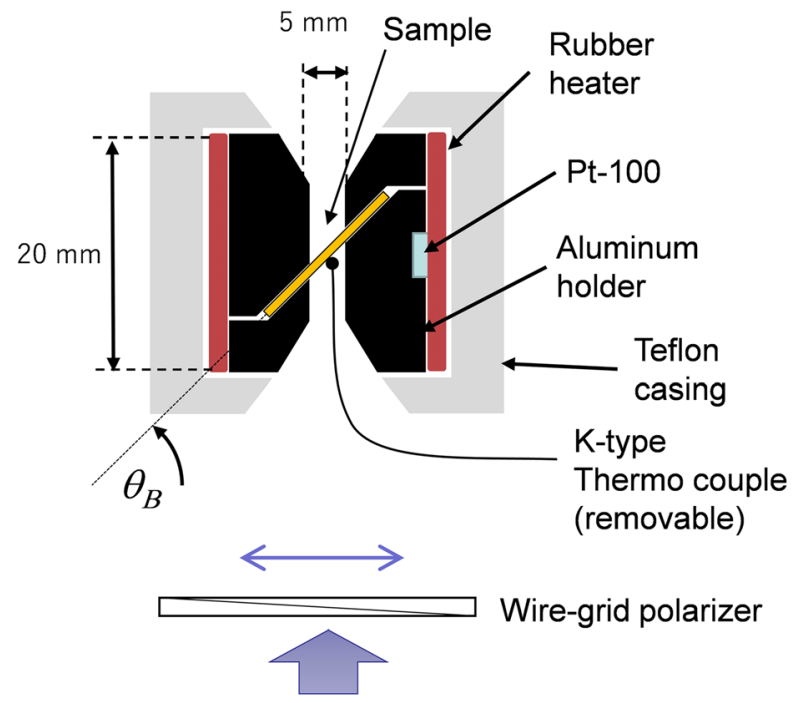

THz beam 
Fig. $3 \mathrm{THz}$ absorption spectrum measured at $30^{\circ} \mathrm{C}$ (upper panel) and its second derivative (lower panel) for a sample A and $\mathbf{b}$ sample B. Arrows in the second derivative spectra show the negative band position

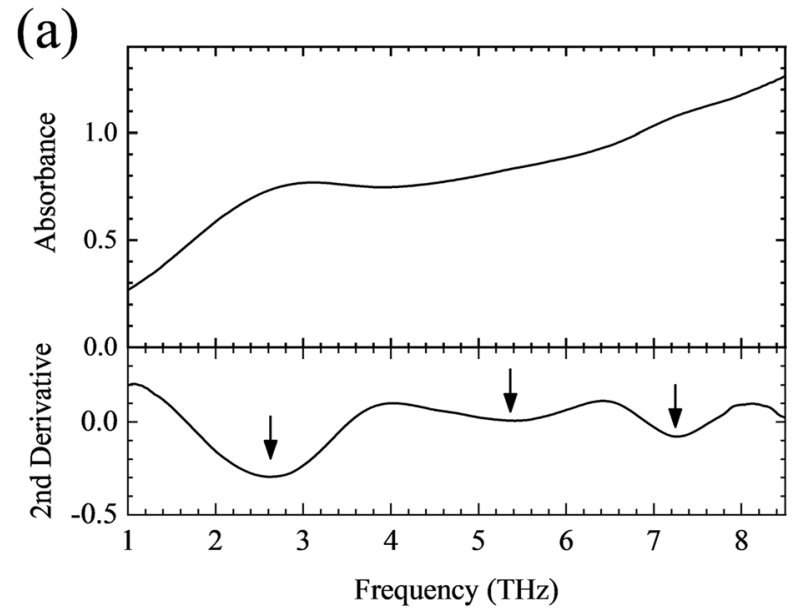

(b)

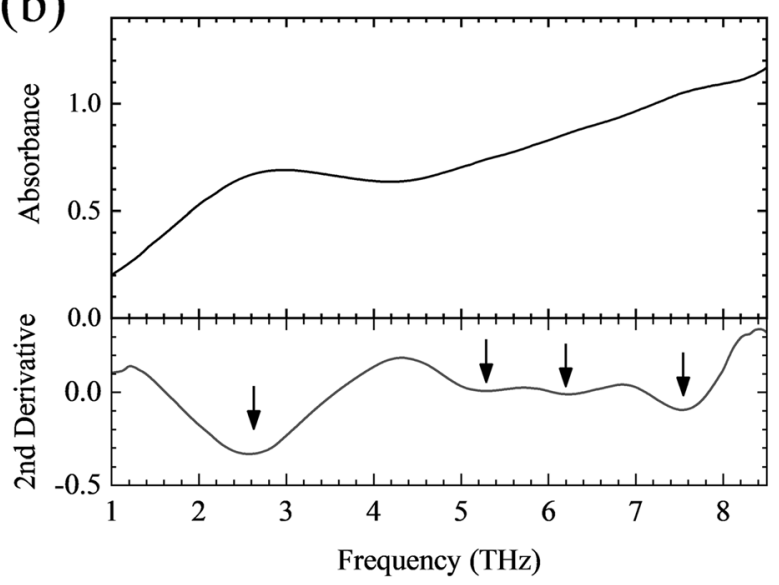

smooth" spectra. Also, smoothing was applied with 100 points when the second derivative spectra was calculated. By taking the second derivative of the absorption intensity with frequency, the broad baseline is removed, and the resulting absorption band structure appears as negative peaks. In addition, the intensity of the peak in the second derivative is proportional to the absorption intensity of the band if the width and shape of the band are the same.

Absorption bands are observed at 2.6, 5.4, and 7.3 THz for sample A, and at 2.6, 5.3, 6.2, and 7.5 THz for sample B. Similar band structures appear at $2.6 \mathrm{THz}$ in both samples. As described in the introduction, the characteristic absorption band for $\mathrm{C}=\mathrm{O} \cdots \mathrm{N}-\mathrm{H}$ hydrogen bonds is observed in this region. Therefore, it is reasonable to assign this band to the $\mathrm{C}=\mathrm{O} \cdots \mathrm{N}-\mathrm{H}$ hydrogen bond vibration. However, the peak frequency is lower than those observed for nylons. This vibrational frequency red-shifting likely correlates to differences in the reduced mass. In nylons, this mode is the coupled vibration of $\mathrm{C}=\mathrm{O}, \mathrm{N}-\mathrm{H}$, and neighboring $\mathrm{CH}_{2}$. [10] In contrast, samples $\mathrm{A}$ and $\mathrm{B}$ contain phenyl groups near the urethane bond, which may increase reduced mass. Another possible culprit is weaker hydrogen bond. Since samples A and B are amorphous polymers, their average intermolecular distance may be longer than that of crystalline nylons, which would weaken hydrogen bond interactions. 


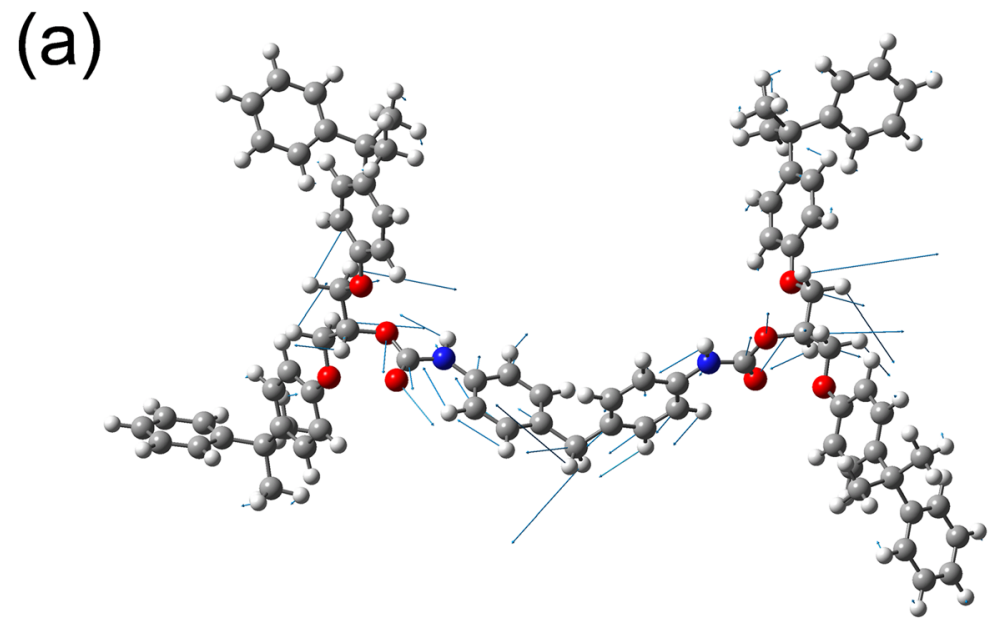

(b)

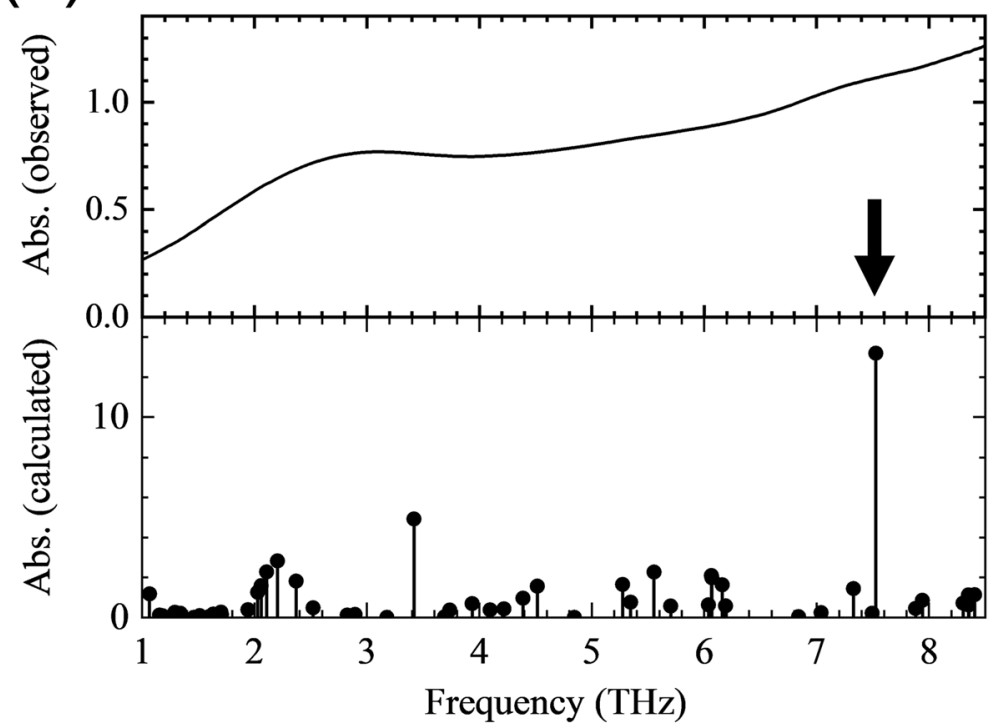

Fig. 4 a Simulation model for molecular vibrations in sample A. The vibrational mode at $7.5 \mathrm{THz}$ is also depicted using vectors. $\mathbf{b} \mathrm{THz}$ absorption spectrum of sample A and vibrational modes obtained from quantum calculations

To assign the other bands, fist principles quantum chemistry calculations were performed. For the lower frequency region of $\mathrm{THz}$ spectra, $\mathrm{THz}$ vibrational modes result mainly from intermolecular motion; a large number of atoms must be included in the simulation. In addition, weak intermolecular interactions must be considered, in order to reproduce anharmonic vibrational potentials. As a result, ab initio calculations for the $\mathrm{THz}$ vibrational spectra require extensive resources compared to those for IR spectroscopy. In the last decade, the frequencies of $\mathrm{THz}$ vibrational modes in crystalline samples have been calculated with some success using software that considers the periodic boundary conditions of the crystal [19-21]. Yamamoto et al. have developed a method (Cartesian-coordinate tensor transfer 
Fig. 5 a Simulation model for molecular vibrations in sample B. The vibrational mode at $7.4 \mathrm{THz}$ is also depicted using vectors. $\mathbf{b} \mathrm{THz}$ absorption spectrum of sample B and vibrational modes obtained from quantum calculations (a)

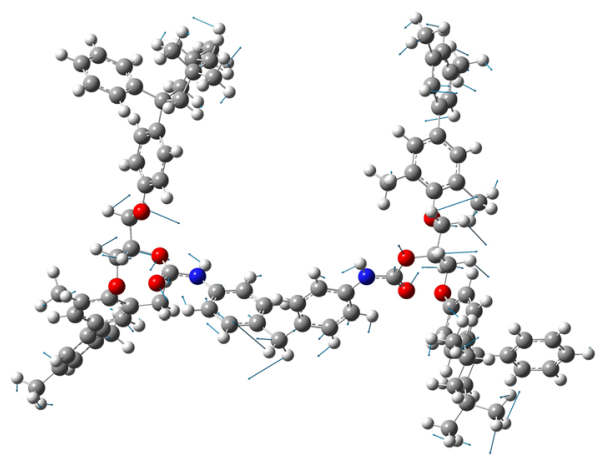

(b)

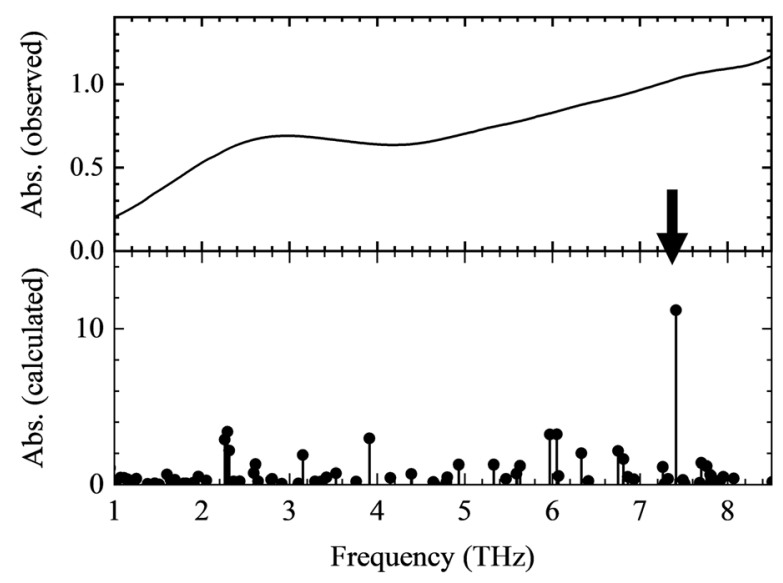

method) to assign polymer spectra using DFT calculations for a fragment of the crystalline polymer [8-10]. However, these methods can be applied only to crystalline samples. To simulate the spectrum of an amorphous structure, more advanced methods, such as ab initio molecular dynamics simulations, are required [22].

Conversely, vibrational modes at higher frequencies (far-IR) are rather straightforward to calculate, because the contribution of intramolecular motion becomes dominant [23, 24]. Some of the bands can be simulated by considering a small number of atoms. Figure 4 a shows a simulation model for sample A, in which MDI bridges two BPAP chains. To extract the model from the actual material, cross-sections of BPAP chains are capped by hydrogen atoms. The geometric structure was optimized and the vibrational frequencies of the simulation model were calculated using the Gaussian 09 program. Optimization and spectrum calculations were executed at the B3LYP/6-311G(d) level. We used the scale factor of 0.966 to compensate the anharmonic effect in the quantum chemistry IR (THz) calculations [25].

Figure $4 \mathrm{~b}$ presents the observed $\mathrm{THz}$ absorption spectrum and calculated vibrational modes for sample A. The majority of the calculated modes are coupled vibrations of many atoms in BPAP and MDI. The obtained frequencies for these modes are not reliable; the frequencies change with the size of the simulation model. On the other hand, the vibrational mode at $7.5 \mathrm{THz}$ is completely intramolecular. Arrows in Fig. 4a demonstrate the atomic motions of that mode. (Also shown as a movie in the supplemental data.) Atoms in the cross-linked 
Fig. $6 \mathrm{THz}$ absorption spectra measured in $5{ }^{\circ} \mathrm{C}$ increments from 30 to $180{ }^{\circ} \mathrm{C}$ (upper panel) and its second derivative (lower panel) a for sample A and $\mathbf{b}$ sample B
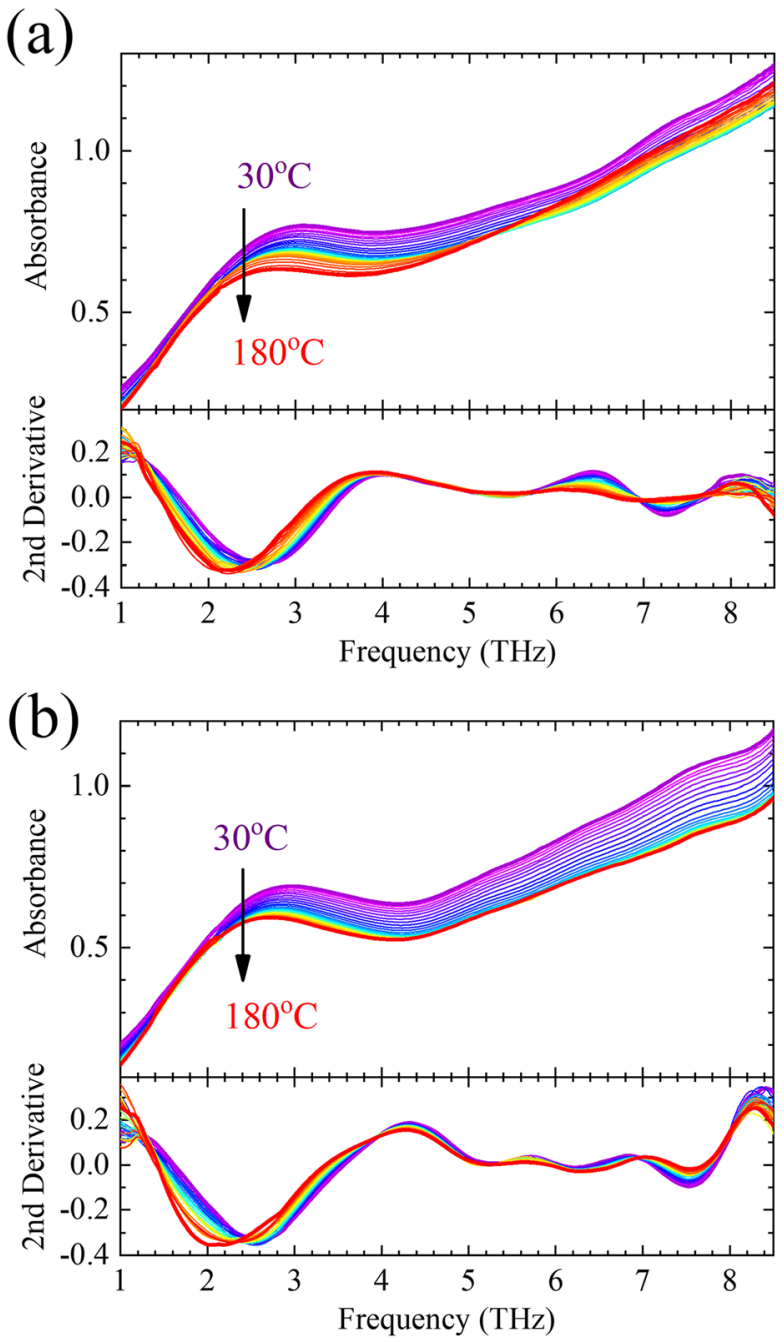

structure, including the urethane bond, move, but the other atoms in the BPAP moiety remain stationary. This mode is completely "local," thus the obtained frequency is reliable. We assigned this band to the torsional vibration motion in the cross-linked structure, coupled with the bending motion of urethane bonds.

Figure 5 a shows a simulation model for sample B, in which MDI bridges BP-TMC-coTMDHB chains. The geometric structure was optimized and the vibrational frequencies were calculated with same method and basis set with sample A, and the scale factor of 0.966 was applied. Figure $5 \mathrm{~b}$ presents the observed $\mathrm{THz}$ absorption spectrum and calculated vibrational modes for sample B. Same as the sample A, the vibrational mode at $7.4 \mathrm{THz}$ is mainly due to intramolecular motion, and we assigned this band to the torsional vibration motion in the cross-linked structure, coupled with the bending motion of urethane bonds.

Figure 6 shows the $\mathrm{THz}$ absorption spectra of samples A and B measured with heating at $5{ }^{\circ} \mathrm{C}$ increments from 30 to $180{ }^{\circ} \mathrm{C}$, as well as their second derivative spectra. The bands at 
Fig. 7 The first derivative of absorption intensity with temperature calculated by the absorption spectra shown in Fig. 6 for (a) sample A and (b) sample B. The dashed lines indicate the frequency shift of the negative peaks
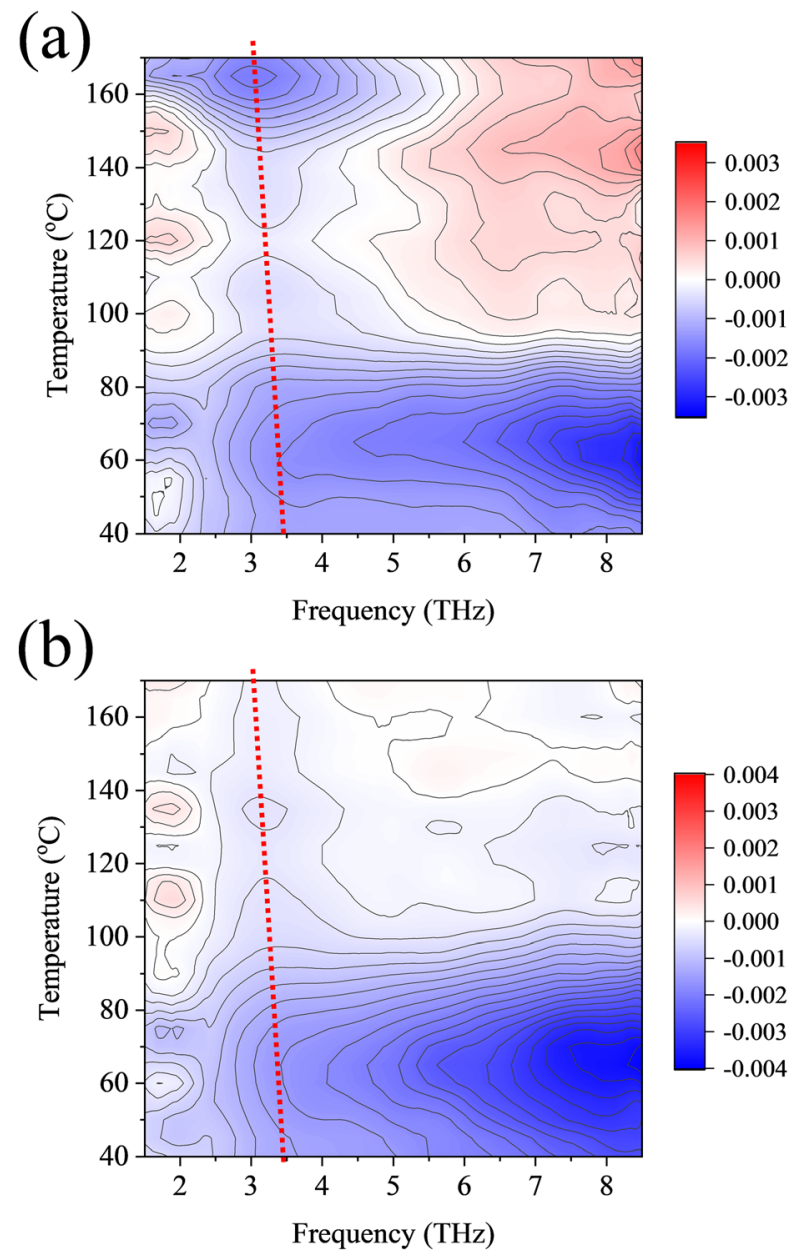

2.6 THz shift toward lower frequencies with temperature monotonically. In our previous studies with crystalline polymers, we observed similar red shifts in the characteristic absorption bands for hydrogen bonds with temperature [7, 9, 14]. The observed rates of frequency shift were approximately $0.1-0.2 \mathrm{THz} / 100{ }^{\circ} \mathrm{C}$ and were attributed to the reduction of intermolecular interactions due to increased intermolecular distances resulting from thermal expansion. The shift in frequency of the $2.6 \mathrm{THz}$ band for samples A and B can also be attributed to reduced intermolecular hydrogen bonds at higher temperatures.

Conversely, the band at $7.5 \mathrm{THz}$ does not exhibit remarkable shifts in frequency with temperature, but the intensity of the second derivative spectrum decreases. This indicates that the static structure shown in Fig. $4 \mathrm{a}$ is not maintained at higher temperatures. Thermal motion likely deforms the structure around the cross-linked molecules, which causes weakening or broadening of the vibrational band.

To clearly visualize the change in absorption intensity, the first derivative of absorption intensity $(\alpha)$ with temperature (T), $\partial \alpha / \partial T$ was calculated. Figure 7 shows two-dimensional plot of $\partial \alpha / \partial T$ with frequency and temperature. The dashed lines in Fig. 7 indicate the frequency 
shift of the negative peaks, which correspond to the hydrogen bond vibration at $2.6 \mathrm{THz}$. Since the band features are tilted by the slope of their baselines, their frequency shift causes a decrease in the absorption intensity. (See Fig. 6) Therefore, a negative peak appears at a slightly higher frequency in the plot of $\partial \alpha / \partial T$. A broad negative band centered at $3 \mathrm{THz}$ appears at $160{ }^{\circ} \mathrm{C}$ only in the plot of sample A, which clearly indicates a change in spectral intensity at the glass transition temperature. A drastic decrease in the intensity of the hydrogen bond vibration occurs at the glass transition temperature. Most probably, hydrogen bonds formed in the glassy structure break when the polymer changes to a rubbery state.

A broad negative area above $3 \mathrm{THz}$ is observed below 90 and $100{ }^{\circ} \mathrm{C}$ for samples $\mathrm{A}$ and $\mathrm{B}$, respectively. Since the bandwidth of this spectral feature is quite broad, this absorption feature may originate with liquid molecules, such as remaining MEK or unreacted MDI. It is difficult to assign molecular structure using the $\mathrm{THz}$ spectra alone. However, DSC, NMR, and dielectric spectroscopy measurements do not indicate corresponding structural changes at these temperatures. The origin of this spectral change remains unknown.

\section{Conclusion}

In this research, THz absorption spectra of cross-linked polyurethane films were observed and peaks in the obtained spectra were assigned using ab initio calculations. A characteristic absorption band at $2.6 \mathrm{THz}$ was assigned to $\mathrm{C}=\mathrm{O} \cdots \mathrm{N}-\mathrm{H}$ hydrogen bond vibration. Another band at $7.5 \mathrm{THz}$ was assigned to the torsional vibration of cross-linked molecules with urethane bonds using DFT calculations. Temperature-dependent spectra and its first derivative spectra clarify slight changes in the broad vibrational bands. Thermal dependence of the absorption intensities indicates the dynamics of hydrogen bond and cross-linked structures in the polyurethane films. Thus, we have demonstrated the potential for $\mathrm{THz}$ spectroscopy to analyze hydrogen bond interactions and cross-linked structures in polymers.

Open Access This article is licensed under a Creative Commons Attribution 4.0 International License, which permits use, sharing, adaptation, distribution and reproduction in any medium or format, as long as you give appropriate credit to the original author(s) and the source, provide a link to the Creative Commons licence, and indicate if changes were made. The images or other third party material in this article are included in the article's Creative Commons licence, unless indicated otherwise in a credit line to the material. If material is not included in the article's Creative Commons licence and your intended use is not permitted by statutory regulation or exceeds the permitted use, you will need to obtain permission directly from the copyright holder. To view a copy of this licence, visit http://creativecommons.org/licenses/by/4.0/.

\section{References}

1. Lee Y-S (2009) Principle of Terahertz Science and Technology. Springer, New York

2. Peiponen K-E, Zeitler A, Kuwata-Gonokami M (2013) Terahertz Spectroscopy and Imaging. Springer Berlin Heidelberg, Berlin, Heidelberg

3. Bershtein V, Ryzhov V (1994) Far infrared spectroscopy of polymers. Polym Anal Charact 114:43-121

4. Wietzke S, Jördens C, Krumbholz N, Baudrit B, Bastian M, Koch M (2007) Terahertz imaging: A new nondestructive technique for the quality control of plastic weld joints. J Eur Opt Soc 2:2-6 . doi: https://doi. org/10.2971/jeos.2007.07013

5. Wietzke S, Jansen C, Reuter M, Jung T, Kraft D, Chatterjee S, Fischer BM, Koch M (2011) Terahertz spectroscopy on polymers: A review of morphological studies. J Mol Struct 1006:41-51 . doi: https://doi. org/10.1016/j.molstruc.2011.07.036 
6. Hoshina H, Ishii S, Yamamoto S, Morisawa Y, Sato H, Uchiyama T, Ozaki Y, Otani C (2013) Terahertz spectroscopy in polymer research: Assignment of intermolecular vibrational modes and structural characterization of poly(3-hydroxybutyrate). IEEE Trans Terahertz Sci Technol 3:248-258 . doi: https://doi. org/10.1109/TTHZ.2013.2253154

7. Hoshina H, Morisawa Y, Sato H, Minamide H, Noda I, Ozaki Y, Otani C (2011) Polarization and temperature dependent spectra of poly(3-hydroxyalkanoate)s measured at terahertz frequencies. Phys Chem Chem Phys 13:9173-9 . doi: https://doi.org/10.1039/c0cp02435j

8. Yamamoto S, Morisawa Y, Sato H, Hoshina H, Ozaki Y (2013) Quantum mechanical interpretation of intermolecular vibrational modes of crystalline poly-(R)-3-hydroxybutyrate observed in low-frequency raman and terahertz spectra. J Phys Chem B 117:2180-2187 . doi: https://doi.org/10.1021/jp309704k

9. Yamamoto S, Miyada M, Sato H, Hoshina H, Ozaki Y (2017) Low-Frequency Vibrational Modes of Poly(glycolic acid) and Thermal Expansion of Crystal Lattice Assigned On the Basis of DFT-Spectral Simulation Aided with a Fragment Method. J Phys Chem B 121:1128-1138 . doi: https://doi.org/10.1021 /acs.jpcb.6b11304

10. Yamamoto S, Ohnishi E, Sato H, Hoshina H, Ishikawa D, Ozaki Y (2019) Low-Frequency Vibrational Modes of Nylon 6 Studied by Using Infrared and Raman Spectroscopies and Density Functional Theory Calculations. J Phys Chem B 123:5368-5376 . doi: https://doi.org/10.1021/acs.jpcb.9b04347

11. Tadokoro H (1968) Structural Studies of Polyesters. II. Far-Infrared Spectra of Aliphatic Polyesters: Comparison with $\alpha$-Polyamides. J Chem Phys 49:3359 . doi: https://doi.org/10.1063/1.1670608

12. Jakeš J, Krimm S (1971) Normal coordinate analyses of molecules with the amide group. Spectrochim Acta Part A Mol Spectrosc 27:35-63 . doi: https://doi.org/10.1016/0584-8539(71)80005-3

13. Matsubara I, Magill J (1973) Lower-frequency infrared spectra and structures of some typical aliphatic polyamides. J Polym Sci Polym Phys Ed 11:1173-1187

14. Suzuki H, Ishii S, Sato H, Yamamoto S, Morisawa Y, Ozaki Y, Uchiyama T, Otani C, Hoshina H (2013) Brill transition of nylon- 6 characterized by low-frequency vibration through terahertz absorption spectroscopy. Chem Phys Lett 575:36-39 . doi: https://doi.org/10.1016/j.cplett.2013.05.002

15. Suzuki H, Ishii S, Otani C, Hoshina H (2015) Low-frequency vibrations of polyamide-6 as a function of temperature and thermal history investigated by terahertz absorption spectroscopy. Eur Polym J 67:284-291 . doi: https://doi.org/10.1016/j.eurpolymj.2015.04.009

16. Hoshina H, Ozaki A, Itagaki Y, Yajima S, Suzuki H, Ishii S, Ishida M, Uchiyama T, Kimura K, Otani C (2014) Sol-gel transition of organogels observed by terahertz spectroscopy. Chem Phys Lett 608:173-176 . doi: https://doi.org/10.1016/j.cplett.2014.05.089

17. Zavodov I a., Kuznetsova LM, Grigorieva SV, Maklakov LI (1996) Far-infrared spectra of some segmented polyurethanes. J Mol Struct 375:193-196 . doi: https://doi.org/10.1016/0022-2860(95)09095-9

18. Hoshina H, Iwasaki Y, Katahira E, Okamoto M, Otani C (2018) Structure and dynamics of bound water in poly ( ethylene-vinylalcohol ) copolymers studied by terahertz spectroscopy. Polymer (Guildf) 148:49-60 . doi: https://doi.org/10.1016/j.polymer.2018.06.020

19. Zhang F, Hayashi M, Wang HW, Tominaga K, Kambara O, Nishizawa JI, Sasaki T (2014) Terahertz spectroscopy and solid-state density functional theory calculation of anthracene: Effect of dispersion force on the vibrational modes. J Chem Phys 140: . doi: https://doi.org/10.1063/1.4873421

20. Jepsen PU, Clark SJ (2007) Precise ab-initio prediction of terahertz vibrational modes in crystalline systems. Chem Phys Lett 442:275-280 . doi: https://doi.org/10.1016/j.cplett.2007.05.112

21. Delaney SP, Witko EM, Smith TM, Korter TM (2012) Investigating tautomeric polymorphism in crystalline anthranilic acid using terahertz spectroscopy and solid-state density functional theory. J Phys Chem A 116: 8051-8057 . doi: https://doi.org/10.1021/jp303961w

22. Ruggiero MT, Krynski M, Kissi EO, Sibik J, Mark1 D, Tan NY, Arslanov D, van der Zande W, Redlich B, Korter TM, Grohganz H, Loebmann K, Rades T, Elliott SR, Zeitler JA (2017) The Significance of the Amorphous Potential Energy Landscape for Dictating Glassy Dynamics and Driving Solid-State Crystallisation. Phys Chem Chem Phys 19:30039-30047 . doi: https://oi.org/10.1039/C7CP06664C

23. Zhang F, Wang HW, Tominaga K, Hayashi M (2016) Mixing of intermolecular and intramolecular vibrations in optical phonon modes: terahertz spectroscopy and solid-state density functional theory. Wiley Interdiscip Rev Comput Mol Sci 6:386-409 . doi: https://doi.org/10.1002/wcms.1256

24. Zhang F, Wang HW, Tominaga K, Hayashi M (2016) Characteristics of Low-Frequency Molecular Phonon Modes Studied by THz Spectroscopy and Solid-State ab Initio Theory: Polymorphs I and III of Diflunisal. J Phys Chem B 120:1698-1710 . doi: https://doi.org/10.1021/acs.jpcb.5b08798

25. Russell D. JohnsonIII (2019) NIST Computational Chemistry Comparison and Benchmark Database. In: NIST Standard Reference Database Number 101

Publisher's Note Springer Nature remains neutral with regard to jurisdictional claims in published maps and institutional affiliations. 